\title{
NUMERICAL SIMULATION OF FORMING PROCESSES, USING AN ARBITRARY LAGRANGIAN EULERIAN FINITE ELEMENT METHOD
}

\author{
A.H. van den Boogaard \\ TNO Building and Construction Research, \\ P.O. box 49, 2600 AA Delft, The Netherlands \\ and \\ J. Huétink \\ University of Twente
}

\begin{abstract}
In this contribution, an Arbitrary Lagrangian Eulerian finite element method is discussed that can be regarded as a continuous remeshing procedure. In the ALE method, the nodal displacements and material displacements are not coupled. This enables the user to keep the mesh regular while the material deforms heavily. The method is well suited for the simulation of processes with very large deformations of solids and path dependent material properties. In the extreme, a steady state process can be simulated, with an Eulerian description in stream direction and at the same time a Lagrangian description perpendicular to the stream direction following the free boundary. The method is demonstrated with an example in the field of roll forming.
\end{abstract}

Key words: finite element method, forming processes, Arbitrary Lagrangian Eulerian method

\section{Introduction}

The simulation of forming processes involves the solution of strongly nonlinear equations. The material behaviour exhibits plasticity and is probably temperature and deformation rate dependent. During the forming process the geometry changes significantly, introducing geometrically nonlinear behaviour. Besides the nonlinearity, the large deformations can cause numerical problems because of heavily distorted meshes.

In a Total Lagrange or Updated Lagrange formulation the material is fixed to the computational grid. This method is very useful if the response of a workpiece is path dependent, such as in the case of plasticity. In case of large plastic deformations however, the computational grid can become too distorted in order to yield correct results. In practice this drawback can be avoided by remeshing techniques. The distorted mesh is substituted by a new more regular mesh. The information that is available in the original mesh must be transferred to the new mesh. In an elasto-plastic analysis this transfer will at least be performed on the stresses and the equivalent plastic strains or the back stress in case of kinematic hardening. These items however are primarily available in the integration points of the elements, i.e. in a limited number of discrete points in the continuum. The integration points of the new mesh will in general not coincide with the integration points of the old mesh. The values of the transferred items can e.g. be 
calculated by defining a smooth function inside the elements and using averaged values in the nodes. This process can be shown to yield some numerical diffusion of the results [1]. If remeshing is applied only a couple of times during the analysis the numerical diffusion may be acceptable, but if the remeshing is to be adopted very often this will deteriorate the results.

In simulations of (semi-)continuous processes such as wire drawing, rolling and extrusion the mechanical deformations take place in an area that is very small compared to the length of the workpiece. In these circumstances one would like to model only the relevant part of the workpiece and let material flow into the mesh at the entrance part and out of the mesh at the exit. This indicates the use of an Eulerian mesh: keep the computational grid fixed in space and let the material flow through it. Two factors complicate this point of view. Firstly the path dependent material behaviour necessitates the integration along streamlines and secondly the boundaries perpendicular to the process flow may not be known in advance. This stimulated several researchers to develop a combination of a Lagrangian and an Eulerian method (e.g. [2] and [3]) for forming processes and [4] for fluid problems). In an Arbitrary Lagrangian Eulerian method the material displacements and the grid displacements are not coupled as in a Lagrangian method and the grid displacement (or velocity) is not equal to zero as in an Eulerian method. In every grid point the material displacements and the grid displacements are now both primary unknowns. The material displacements are determined by the constitutive and kinematic relations and the boundary conditions. The grid displacements are determined in a way to maintain a physically and computationally proper grid, i.e. keeping the information about material boundaries and introducing a less distorted mesh. Basically two types of Arbitrary Lagrangian Eulerian methods can be formulated considering the way in which the grid displacements are calculated. The first type utilizes a global definition, e.g. the solution of a differential equation or minimization of a functional, to determine the 'optimal' grid displacements [3]. The control over the element mesh is based on a mathematical description that might be appropriate for a number of geometries. A disadvantage of this method is that it doubles the number of unknowns that has to be solved simultaneously. The second type utilizes an explicit prescription of the grid displacement depending on other grid and material displacements [2]. Here for every problem a specific prescription for the grid displacements must be specified, the number of unknowns however remains equal to the number of unknowns in an ordinary Lagrangian (or Eulerian) method.

In the next section the basic ALE equation is derived in infinitesimal form. Then two possible ways to integrate this equation are discussed that could be used in a finite element code. One of the methods is chosen and the advantage over the other method is indicated. In section 3 an example is shown of a profile rolling process, in which the described method is used to simulate a material flow through the mesh. The final section indicates some first conclusions and the developments that take place now and in the near future.

\section{A continuous remeshing technique}

The ALE method is easily presented in infinitesimal form, based on velocities rather than on displacements. The velocity of a material point $\mathbf{x}^{m}$ at time $t$ is defined by 


$$
\mathbf{v}^{m}=\frac{\mathrm{d} \mathbf{x}^{m}}{\mathrm{~d} t}=\dot{\mathbf{x}}^{m}
$$

and the velocity of a grid point by

$$
\mathbf{v}^{g}=\frac{\mathrm{d} \mathbf{x}^{g}}{\mathrm{~d} t}=\dot{\mathbf{x}}^{g}
$$

If we consider a material related quantity $\phi$, like a stress or the equivalent plastic strain, we can calculate the rate of change with respect to the material and to the grid point.

$$
\begin{aligned}
& \dot{\phi}^{m}=\frac{\partial \phi}{\partial t}+\dot{\mathbf{x}}^{m} \cdot \nabla \phi \\
& \dot{\phi}^{g}=\frac{\partial \phi}{\partial t}+\dot{\mathbf{x}}^{g} \cdot \nabla \phi
\end{aligned}
$$

From (3) and (4) the basic equation for an Arbitrary Lagrangian Eulerian algorithm to calculate the rate of change for a grid point can be derived.

$$
\dot{\phi}^{g}=\dot{\phi}^{m}+\left(\dot{\mathbf{x}}^{g}-\dot{\mathbf{x}}^{m}\right) \cdot \nabla \phi
$$

The first item in the right hand side of (5) represents the material rate of change, like in an Updated Lagrange analysis, the second part represents the convective rate of change. In a general purpose finite element program we would like to use the algorithms that are already derived for the constitutive behaviour in an Updated Lagrange analysis. For this purpose we can choose to integrate (5) independently for the material derivative part and for the convective part. Two schemes are now possible: first the material and then the convective part or first the convective and then the material part. In figure 1 the schemes are represented graphically. The first scheme is presented by the solid lines and the second by the dashed lines.

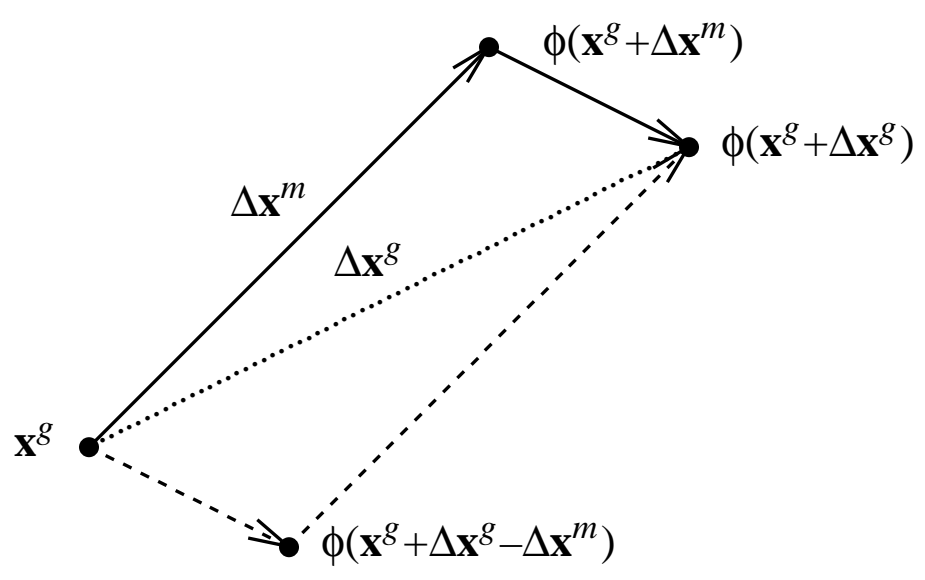

Figure 1: Integration paths for the basic ALE equation.

In formulas the two different schemes can be presented as:

$$
\phi\left(\mathbf{x}^{g}+\Delta \mathbf{x}^{g}, t+\Delta t\right)=\phi\left(\mathbf{x}^{g}, t\right)+\Delta \phi^{c}(t+\Delta t)+\Delta \phi^{m}\left(\mathbf{x}^{g}\right)
$$


for the 'first material and then convective' scheme and

$$
\phi\left(\mathbf{x}^{g}+\Delta \mathbf{x}^{g}, t+\Delta t\right)=\phi\left(\mathbf{x}^{g}, t\right)+\Delta \phi^{c}(t)+\Delta \phi^{m}\left(\mathbf{x}^{g}+\Delta \mathbf{x}^{g}-\Delta \mathbf{x}^{m}\right)
$$

for the 'first convective and then material' scheme. The convective increment $\Delta \phi^{c}$ is determined by:

$$
\Delta \phi^{c}(t+\Delta t)=\phi\left(\mathbf{x}^{g}+\Delta \mathbf{x}^{g}, t+\Delta t\right)-\phi\left(\mathbf{x}^{g}+\Delta \mathbf{x}^{m}, t+\Delta t\right)
$$

for the first scheme and

$$
\Delta \phi^{c}(t)=\phi\left(\mathbf{x}^{g}+\Delta \mathbf{x}^{g}-\Delta \mathbf{x}^{m}, t\right)-\phi\left(\mathbf{x}^{g}, t\right)
$$

for the second.

If we substitute (8) and (9) in (6) and (7) some items in the right hand side cancel or are even equal to the left hand side. Nevertheless the formulas are presented this way because they show the grid values $\phi\left(\mathbf{x}^{g}, t\right)$ and $\Delta \phi^{m}$ that are also present in the ordinary Updated Lagrangian method and the previously absent convective increments $\Delta \phi^{c}$. These presentations of the formulas clearly shows the similarities and differences between the Lagrangian and the Arbitrary Lagrangian Eulerian method. For both methods the crux is the calculation of the convective parts $\phi\left(\mathbf{x}^{g}+\Delta \mathbf{x}^{g}, t+\Delta t\right)-\phi\left(\mathbf{x}^{g}+\Delta \mathbf{x}^{m}, t+\Delta t\right)$ or $\phi\left(\mathbf{x}^{g}+\Delta \mathbf{x}^{g}-\Delta \mathbf{x}^{m}, t\right)-\phi\left(\mathbf{x}^{g}, t\right)$. As can be appreciated from figure 1 the determination of the convective increment for both schemes is equivalent, when their respective values of $\mathbf{x}^{g}, \Delta \mathbf{x}^{g}, \Delta \mathbf{x}^{m}, t$ and $t+\Delta t$ are substituted. In the sequel only the first scheme is elaborated. The method is based on the elaboration of Huétink et.al. [5] for the second scheme, a weighed local global smoothing.

First local smoothing is applied in which the integration point values for $\phi$ are extrapolated to the nodes and then the value of $\phi$ in a node is determined by averaging the contribution of the connected elements; the global smoothing (see e.g. [6]). The nodal values of $\phi$ are collected in a vector $\boldsymbol{\Phi}$. We now have obtained a continuous field $\phi$, determined by the nodal point values $\Phi$ and the element interpolation functions $\Psi(\xi)$. The values of $\boldsymbol{\Psi}$ only depend on the element shape and the (isoparametric) coordinates inside the element.

With this global $\phi$-field at hand it is tempting to directly derive the new grid point values from

$$
\phi\left(\mathbf{x}^{g}+\Delta \mathbf{x}^{g}, t+\Delta t\right)=\Psi^{\mathrm{T}}\left(\xi^{g}+\Delta \xi^{g}\right) \boldsymbol{\Phi}(t+\Delta t) .
$$

This procedure leads however to significant numerical diffusion. After a number of successive local global smoothing operations high gradients in $\phi$ will weaken.

Alternatively the global $\phi$-field can be used to only determine the convective part of equation (6).

$\phi\left(\mathbf{x}^{g}+\Delta \mathbf{x}^{g}, t+\Delta t\right)=\phi\left(\mathbf{x}^{g}, t\right)+\Delta \phi^{m}\left(\mathbf{x}^{g}\right)+\left[\Psi\left(\xi^{g}+\Delta \xi^{g}\right)-\Psi\left(\xi^{g}+\Delta \xi^{m}\right)\right]^{\mathrm{T}} \boldsymbol{\Phi}(t+\Delta t)$

This procedure can cause numerical instabilities depending on the convection size $\Delta \mathbf{x}^{g}-\Delta \mathbf{x}^{m}$ relative to the element size $h$. This ratio is known as the Courant number $C$.

$$
C=\frac{\Delta \mathbf{x}^{g}-\Delta \mathbf{x}^{m}}{h}
$$


It is known that for a Galerkin discretisation the Courant number should not exceed the value of 0.5 for reasons of stability (see e.g. [7], p.445).

To compromise between both approaches a weighing procedure is used in which the value of $\phi\left(\mathbf{x}^{g}+\Delta \mathbf{x}^{g}\right)$ is calculated partly from the smoothed $\phi$-field from equation (10) and partly from equation (11). If we choose a factor $\alpha$ for the smoothed field and $1-\alpha$ for the other field and note that $\phi\left(\mathbf{x}^{g}, t\right)+\Delta \phi^{m}\left(\mathbf{x}^{g}\right)=\phi\left(\mathbf{x}^{g}+\Delta \mathbf{x}^{m}, t+\Delta t\right)$ we get

$$
\begin{aligned}
\phi\left(\mathbf{x}^{g}+\Delta \mathbf{x}^{g}, t+\Delta t\right)= & \boldsymbol{\Psi}^{\mathrm{T}}\left(\xi^{g}+\Delta \xi^{g}\right) \boldsymbol{\Phi}(t+\Delta t)+ \\
& \left.(1-\alpha)\left\{\phi\left(\mathbf{x}^{g}+\Delta \mathbf{x}^{m}, t+\Delta t\right)-\boldsymbol{\Psi}^{\mathrm{T}}\left(\xi^{g}+\Delta \xi^{m}\right)\right]^{\mathrm{T}} \boldsymbol{\Phi}(t+\Delta t)\right\} .
\end{aligned}
$$

The part between parentheses represents the difference between the globally smoothed field and the local value of $\phi$. It can be argued that for small convective steps equation (11) can prevail $(\alpha \ll 1)$, preventing too much numerical diffusion, while for larger convective steps equation (10) prevails $(\alpha \approx 1)$, preventing numerical instabilities. For this reason the weight factor $\alpha$ is made a function of the Courant number $C$. In the presented example in the next section a simple linear relation $\alpha=1.5 C$ is used.
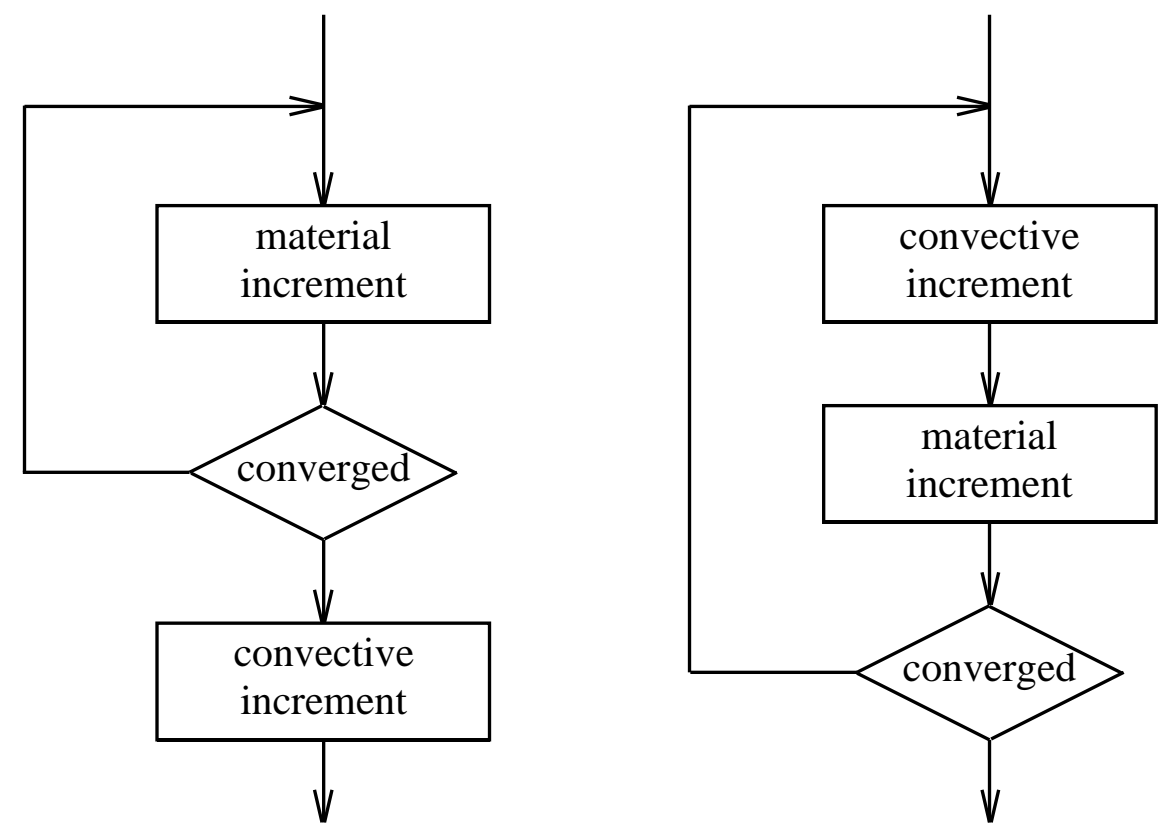

Figure 2: Two possible implementations of an ALE method for structural mechanics.

Having chosen the 'first material then convection' scheme offers the potential of an efficient implementation of the ALE method in an existing finite element code. Because the first step (the Updated Lagrange step) is not influenced by the convective step, we can choose to perform the full equilibrium iterations first on the material displacements and end the increment by one convection step. This introduces an unbalance after the converged equilibrium iterations, but this unbalance is not different from the unbalance that would otherwise be introduced at the start of every iteration. For the "first convection 
then material' scheme this solution method is not possible since the convective increment is influenced by the displacements of the 'material' part of the iteration $\left(\Delta \mathbf{x}^{m}\right.$ in equation 9 ). Both schemes are shown in figure 2. The chosen scheme (left in figure 2) has the advantage that the main part of the algorithm is based on an ordinary Updated Lagrange algorithm. The original software including the (consistent) tangential stiffness matrices are unaltered. The algorithm can be interpreted as an ordinary Updated Lagrange method with a remeshing procedure after every increment, outside the iteration loop, therefore the name continuous remeshing technique is suggested.

\section{Example: Roll forming}

In this section the described algorithm is used for the simulation of a part of a roll forming process. In the considered process a hat shaped profile is produced from flat sheet metal. The flange of the profile remains in the original plane of the sheet while the hat shape is formed see figure 3 .

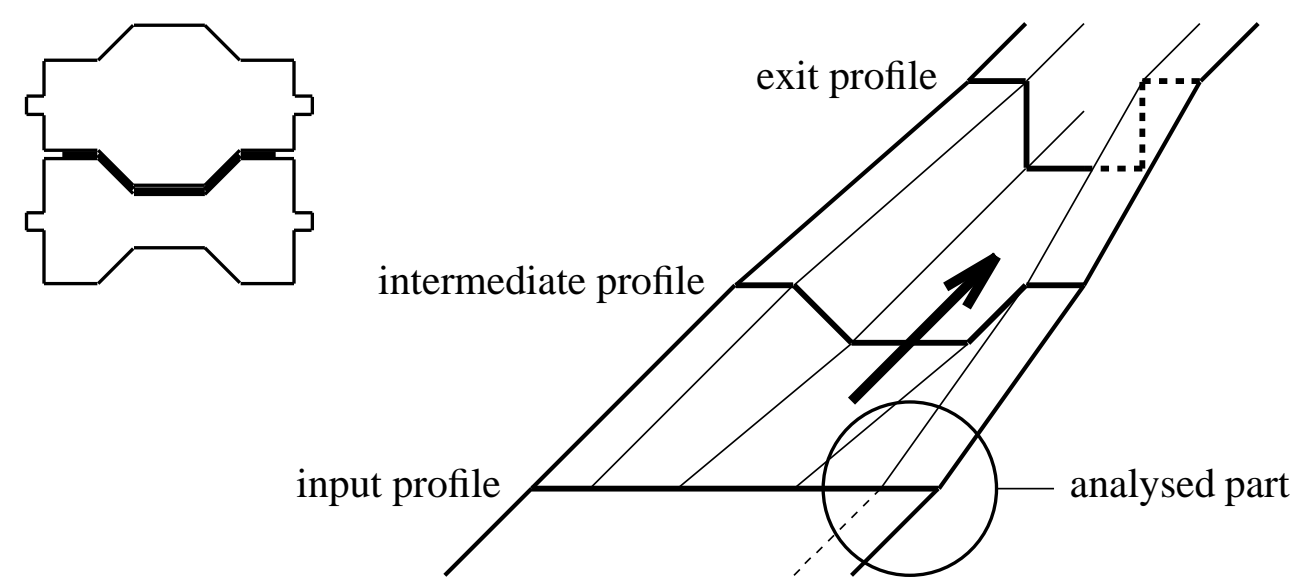

Figure 3: Roll forming of a hat shaped profile in three stands (second stand shown left).

In figure 3 the flat sheet enters at the lower side and the hat shaped profile exits at the upper right corner, the rolls of the second stand are drawn at the left part of the picture. In figure 3, three profiles are plotted, representing the profile at three different positions. The deformations are supposed to take place at three roll stands. In reality there may be more stands, but this does not influence the part that is analysed here. The consequence of the fact that the flange remains in its original plane is that the metal in the flange is deformed in its own plane. A representative part of the flange at the first stand is modeled. It is indicated in figure 3 by the circle. Here the input angle of the flange is $0^{\circ}$ and at the output the angle with the process direction is $1^{\mathrm{O}}$.

In figure 4 the finite element mesh is shown. The mesh consists of 322 four node plane stress elements and 390 nodes. The boundary conditions at the left, top and right edge are imposed such that the velocity in the process direction is equal (prescribed displacements) and the displacements perpendicular to the top edge are suppressed. The used material is steel with a Youngs' modulus of $200000 \mathrm{MPa}$ and plasticity based on the 
Nadai relation:

$$
\sigma_{y}=D\left(\varepsilon_{p 0}+\varepsilon_{p}\right)^{n}
$$

with $D=560 \mathrm{MPa}, \varepsilon_{p 0}=0.005$ and $n=0.2$ and $\varepsilon_{p}$ being the equivalent plastic strain. The initial thickness of the sheet is $0.6 \mathrm{~mm}$ and the width of the flange is $10 \mathrm{~mm}$.

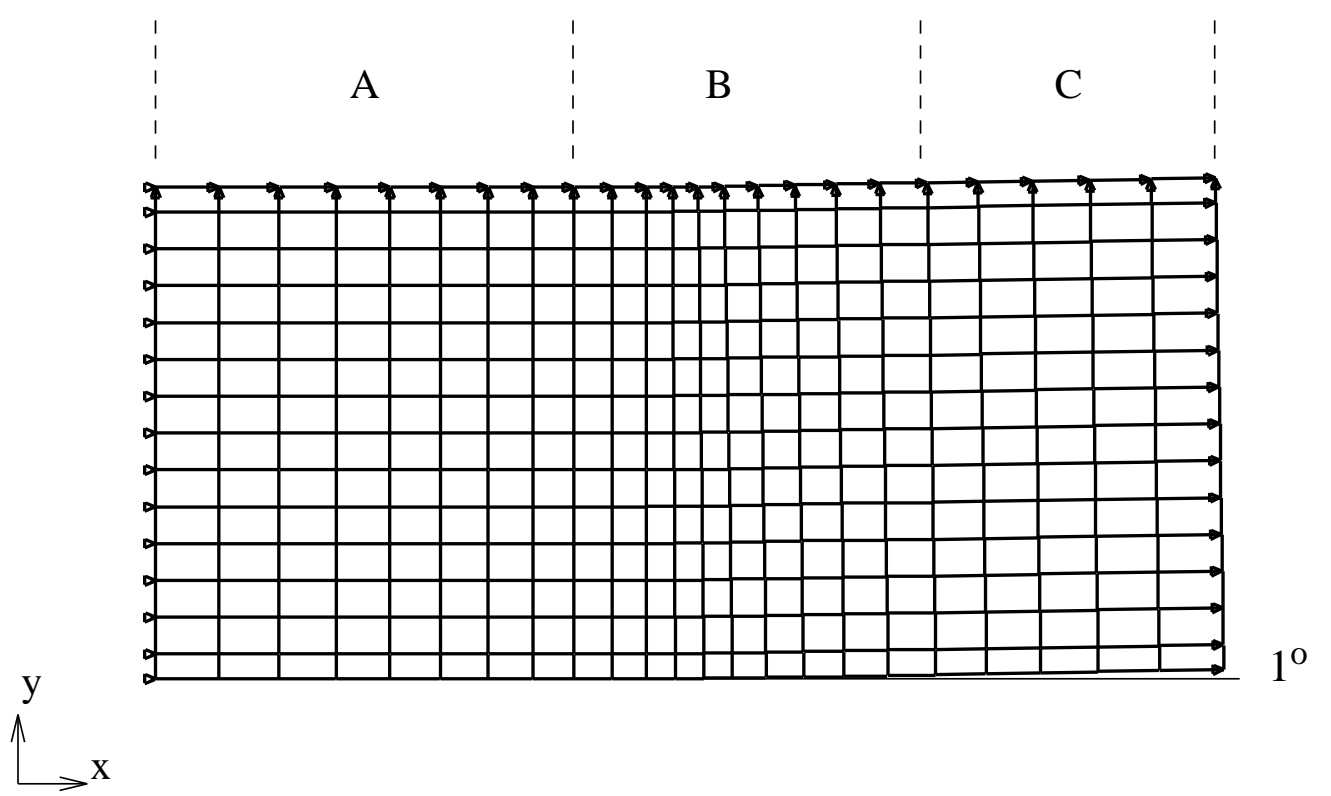

Figure 4: Element mesh of the flange at the first stand.

In every increment the sheet is displaced $0.2 \mathrm{~mm}$ to the right. This results in a maximum Courant number of 0.3 . After an increment the mesh is replaced to its original position, yielding in fact an Eulerian description. This was continued until a steady state solution was reached. After 100 increments the situation in the flange did not change significantly anymore. This is exactly the increment where the material that was at the entrance at the start of the analysis has moved to the exit position. Typically 2 iterations were needed in every increment to comply with an energy convergence criterion of $10^{-4}$.

The results for the calculation of the equivalent plastic strain after 140 increments are presented in figure 5. In figures 6, 7 and 8 the stresses in $x$ - and $y$-direction and the shear stress are plotted. It can be seen that at the entrance (zone A in figure 4) in plane bending dominates. The stresses $\sigma_{x x}$ vary over the height and are tensile at the outer side and compressive at the inner side. The stresses $\sigma_{y y}$ and $\tau_{x y}$ are almost equal to zero at the entrance. The mean $\sigma_{x x}$ at the entrance is tensile and at the exit (zone C) it is compressive. Obviously the plastic deformation in zone B has caused an elongation that has to be corrected in zone $\mathrm{C}$. At the exit the in plane bending moment has changed its sign. Again this can be explained by a high plastic elongation at the outer side of the flange, that has to be corrected in order to let the flange uniformly exit at an angle of $1^{\mathrm{o}}$. In zone $\mathrm{B}$ a shear band develops as can most clearly be seen in figure 8 . The predominant 
Figure 5: Isolines for equivalent plastic strain in steady state situation.

Figure 6: Isolines for $\sigma_{x x}$ in steady state situation.

type of deformation seems to be the shear deformation. In the area where the equivalent plastic strain increases rapidly, the stresses in $x$ - and $y$-direction are small and the shear stresses are at maximum. The plot of $\sigma_{y y}$ indicates that at the entrance the center part of the sheet pulls the flange inside (up), while at the exit the center part pushes the flange outside (down). This differs from static bending where the stresses $\sigma_{y y}$ would be compressive in zone $\mathrm{B}$ and tensile in zone $\mathrm{A}$ and $\mathrm{C}$.

\section{Conclusions and Current Developments}

It is demonstrated that an ALE method, based on a continuous remeshing technique can analyse the process conditions in metal forming processes. The method is potentially more efficient than a fully coupled ALE analysis or a staggered analysis inside the equilibrium iteration loop.

The current developments aim at the analysis of contact conditions in an ALE environment like in [8] and the analysis of thermo-mechanically coupled problems like in [8] and [9]. 
Figure 7: Isolines for $\sigma_{y y}$ in steady state situation.

Figure 8: Isolines for $\tau_{x y}$ in steady state situation.

All presented work was carried out within a development version of DIANA at TNO Building and Construction Research.

\section{Acknowledgements}

The first ALE algorithms in DIANA were programmed and evaluated by P.N. van der Helm during his graduation work after D.G. Roddeman had made the ALE framework available. The first author continues their work and is supported by TNO and the DIANA Users Association. The roll forming example was made as part of a dutch IOP-metals project for the 'Stichting Koudprofileurs' in cooperation with G.J.J. Streefland and E. Langerak of TNO Metals Research Institute. 


\section{References}

[1] J. Huétink, 'Analysis of metal forming processes based on a combined EulerLagrangian FE formulation', in: Numerical Methods in Industrial Forming Processes, (eds. J.F.T. Pittman et.al.), Pineridge Press, Swansea, 501-509, 1982.

[2] J. Huétink and P.N. van der Helm, 'On Euler-Lagrange finite element formulation in forming and fluid problems', in: Numerical Methods in Industrial Forming Processes, (eds. J.-L. Chenot, R.D. Wood, O.C. Zienkiewicz), A.A. Balkema, Rotterdam, 45-54, 1992.

[3] P.J.G. Schreurs, F.E. Veldpaus and W.A.M. Brekelmans, 'Simulation of forming processes, using the arbitrary Eulerian-Lagrangian formulation', Comp. Meth. Appl. Mech. Eng., 58, 19-36, 1986.

[4] T.J.R. Hughes, W.K. Liu, T.K. Zimmerman, 'Lagrangian-Eulerian finite element formulation for incompressible viscous flows', Comp. Meth. Appl. Mech. Eng., 29, 329-349, 1981.

[5] J. Huétink, P.T. Vreede, J. van der Lugt, 'Progress in mixed Eulerian-Lagrangian finite element simulation of forming processes', Int.J.Num.Meth.Eng., 30, 1441-1457, 1990.

[6] E. Hinton and J.S. Campbell, 'Local and global smoothing of discontinuous finite element functions using least square method', Int.J.Num.Meth.Eng., 8, 461-480, 1974.

[7] O.C. Zienkiewicz and R.L. Taylor, 'The finite element method', Vol.2, McGrawHill Book Company, London etc., 1991.

[8] J. van der Lugt, 'A finite element method for the simulation of of thermomechanical contact problems in forming processes', Dissertation, University of Twente, The Netherlands, 1988.

[9] J. Huétink, 'On the simulation of thermo-mechanical forming processes', Dissertation, University of Twente, The Netherlands, 1986. 
i
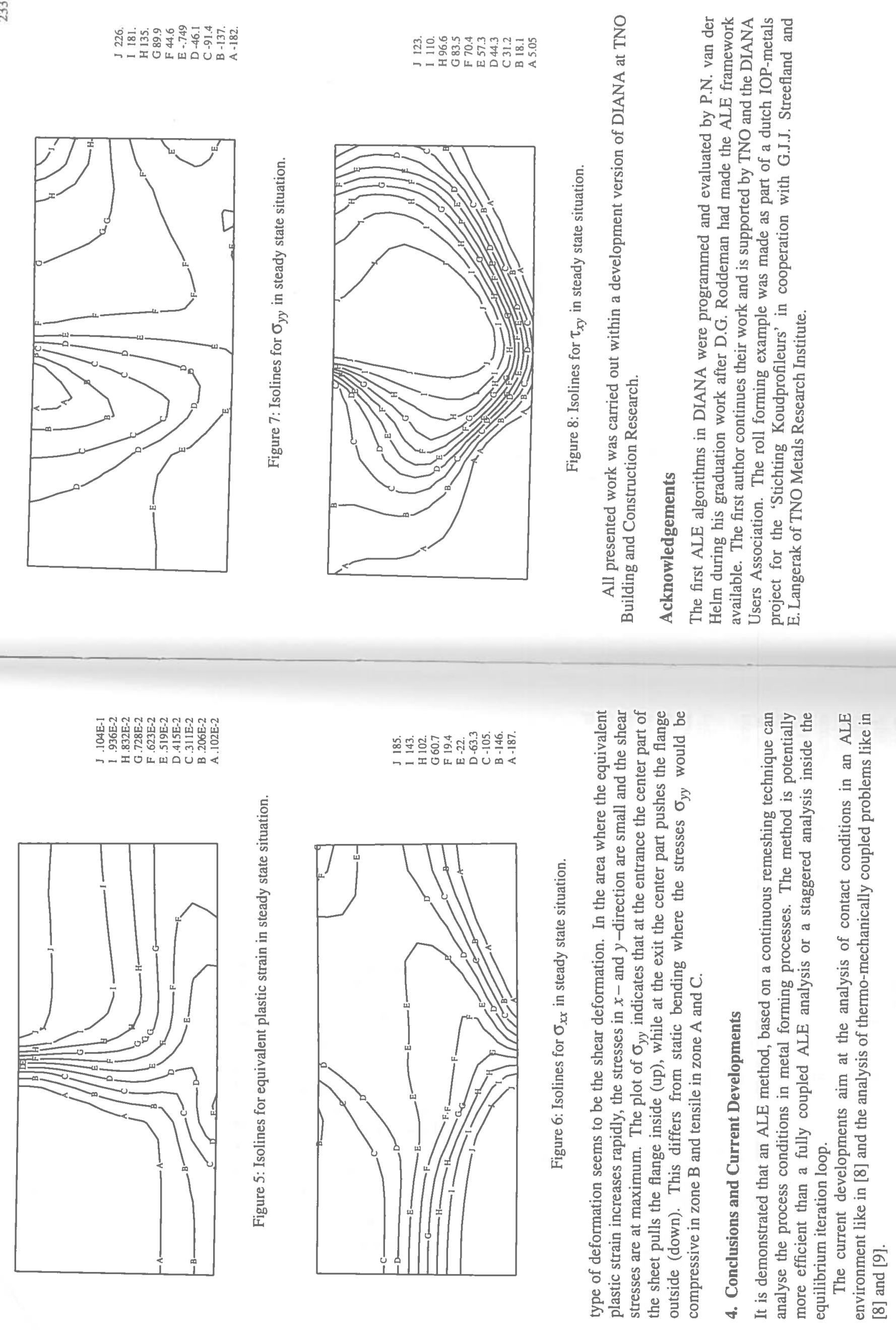